\title{
El turismo creativo. Conceptualización y características
}

\section{Creative tourism. Conceptualization and characteristics}

\author{
Fabrizio Augusto Alberca Sialer* \\ Instituto de Investigación de la Escuela Profesional de Turismo y Hotelería, \\ Universidad de San Martín de Porres, Perú
}

\section{Resumen}

Se reúne conceptos, características e ideas sobre el turismo creativo, que es una derivación del turismo cultural, en el cual el turista se acerca fundamentalmente al patrimonio cultural inmaterial mediante la cocreación. El turismo creativo responde a los tiempos actuales (posmodernidad), pues le permite al turista vivir experiencias únicas, «diferenciadas», no masivas, que también benefician de diversas maneras al anfitrión.

Palabras clave: turismo creativo; turismo cultural; patrimonio cultural inmaterial; posturismo; sostenibilidad

\begin{abstract}
This article gathers concepts, characteristics and ideas about creative tourism: a derivation from cultural tourism in which tourists fundamentally approach intangible cultural heritage through co-creation. Creative tourism responds to current times (postmodernity) because it allows tourists to live unique, «differentiated», non-massive experiences that also benefit the host in various ways.
\end{abstract}

Keywords: creative tourism; cultural tourism; intangible cultural heritage; posttourism; sustainability

Este es un artículo Open Access bajo la licencia Creative Commons Atribución-NoComercial-Compartirlgual 4.0 


\section{Introducción}

La posmodernidad es el marco en el que se insertan las prácticas turísticas actuales. El turista de hoy busca diferenciarse y vivir experiencias únicas. Por consiguiente, este turismo en la posmodernidad (posturismo) se caracteriza por el rechazo al turismo de masas (Urry, 2004), donde no hay diferenciación y el viajero es reducido a un todo sin individualidad. Para Molina, «el posturismo representa una ruptura con el modelo tradicional de turismo, una alternativa que permite el entendimiento del turismo sea práctica o teóricamente a la luz de una nueva sociedad cuyos deseos cambiaron a lo largo del tiempo» (como se citó en Bezerra, Melo, \& Rocha, 2012, p. 1277).

Los posturistas tienen como característica distintiva que «en vez de comprometerse en una vana búsqueda de lo extraño y de lo exótico, viajan en pos de experiencias distintivas pero familiares» (Cohen, 2005, p. 16). Es un viajero que experimenta, pero que no profundiza demasiado (en la intención de conocer la cultura local) en su búsqueda de experiencias, pues le interesa más lo fantasioso y espectacular para vivirlo y publicarlo, buscando una distinción social, dejando de lado la autenticidad (Cohen, 2005).

Se vive, como dice Poon, un «nuevo turismo» de nuevos y más segmentos, flexible y a medida (como se citó en Urry, 2004, p. 19), donde toda manifestación de la cultura es potencialmente un recurso turístico.

El turismo creativo como producto turístico es consecuencia de los tiempos que discurren (además de una expresión del posmodernismo), pues en él se ofrece la cultura «auténtica» y en contraparte el turista experimenta, hace, prueba, crea actividades que son muy personales, las mismas que justamente el posturista busca. Las experiencias, dicen Pine y Gilmore, son herramientas diferenciales de gestión (como se citó en De Freitas, De Sevillha, \& Aráujo, 2018), es decir, procurar experiencias, bien podría ser el enfoque de gestión a seguir.

Una mirada global del tema, nos permite apreciar que los destinos, dadas las condiciones y la realidad, deben convertirse en espacios creativos capaces de dar respuesta a este nuevo turismo y sus nuevos viajeros, cuando no también a sus propios ciudadanos. En ese sentido, en la United Nations 
Conference on Trade and Development (UNCTAD) se plantea que los territorios creativos deben contemplar cuatro sectores ${ }^{1}$ : el patrimonio cultural, las artes, los medios y las creaciones funcionales (como se citó en Cavalcanti, Medeiros de Araujo, \& Guimarães, 2018).

Por ende, el turismo basado en el patrimonio cultural y dispuesto para la creatividad del visitante es una oportunidad para países como el Perú, porque al posturista le interesa esta combinación de actividades y actores, y porque el turismo cultural, en general, es un segmento amplio y en crecimiento: en 2018, el 39\% de los visitantes tuvo motivaciones exclusivamente culturales (World Tourism Organization, 2018a). No obstante, quienes practican turismo de naturaleza, aventura $u$ otros, también suelen realizar un turismo cultural indirecto, ya sea al comer, visitar la ciudad u otras prácticas.

El turismo creativo se ha venido trabajando en países como Nueva Zelanda, Austria, España, Canadá, Estados Unidos y Taiwán (Richards, 2011), es decir, no es un tema nuevo; por ello, se pretende redirigir la mirada hacia él, pensándolo como una oportunidad para la realidad peruana.

Por lo tanto, se plantea como objetivo en este estudio, exponer una revisión de los principales conceptos e ideas sobre el turismo creativo, entendido como una derivación del turismo cultural. Para tal fin, se ha navegado por temas como el posturismo, el turismo cultural, la relación entre turismo y patrimonio, $\mathrm{y}$, finalmente, turismo creativo.

\section{Turismo cultural}

El turismo se perfila como el gran fenómeno social de mediados del siglo pasado e inicios del presente. Ha pasado de contabilizar 25 millones de viajeros en 1950 a 1323 millones de desplazamientos en el año 2017, o un 7\% más de arribos respecto del 2016 (World Tourism Organization, 2018b). Visto de otro modo, cada vez más personas deciden desplazarse para conocer y reconocerse, pues «el conocimiento adquirido cuando se viaja a un país [o

1 El texto original es el siguiente: A noção de territórios criativos contempla quatro setores, a saber: (1) patrimônio cultural; (2) artes (artes visuais e artes dramáticas); (3) mídias (edição e mídia impressa, audiovisual) e (4) criações funcionais (design, moda, novas mídias, arquitetura, consultoria e propaganda (UNCTAD, como se citó en Cavalcanti et al., 2018, p. 278). 
región] diferente al de origen o residencia coadyuva en la valoración tanto de lo propio como de lo foráneo» (Quesada, 2010, p. 98).

Es un hecho incontestable que al hablar de turismo estamos hablando de una de las dimensiones más importantes de la cultura del hombre. El individuo se cultiva viajando y al revalorizarse a sí mismo a fuerza de viajar, se motiva aún más por entrar en contacto con otras culturas, paisajes y contextos. (Valdez, 2002, p. 85)

El turismo, por sí mismo, puede ser motor para el aumento de los viajes al buscar repetir las experiencias vividas, además de ser capaz de enriquecer culturalmente a las personas anfitrionas, quienes pueden aprender al ver otras formas de vida, más aún cuando la diferencia cultural es marcada; no obstante, el aprendizaje cultural del anfitrión no se dará necesariamente con la misma intensidad en todos los casos.

En el pasado uno de los motivos de viaje fue el cultural, por ejemplo, el Grand Tour, ese viaje iniciático y educativo del siglo XVII que bien podría ser considerado el acto de nacimiento del turismo moderno. Empero, si se busca más atrás en el tiempo, se tendrá que ya los griegos viajaban, entre otros lugares, a Olimpia y Delfos, y los romanos a Egipto y Tierra Santa (Khatchikian, 2000).

El turismo es una actividad netamente cultural, sin importar la motivación: «el turismo es sólo una faceta de la dimensión cultural del hombre [...] y por lo tanto todo turismo es de alguna forma necesariamente cultural» (Valdez, 2002, p. 83), razón por la cual la denominación de turismo cultural podría ser considerada redundante. Sin embargo, pese a que hay una motivación de índole cultural subyaciendo a todo movimiento turístico, por motivos operativos y de estudio, se ha propuesto diferentes clasificaciones o tipologías según determinados criterios. Definir las tipologías -con referencia a los turistas- es importante, según lo planteado por Quesada (2010):

Para conocer sus peculiaridades y expectativas de consumo. ¿Para qué nos sirve esto?, con base en esta información, podremos saber con mayor exactitud qué podemos ofrecerle a cada uno de los grupos que conforman lo que llamamos segmentos de mercado. (p. 95) 
Según las motivaciones del visitante, el turismo se clasifica en: turismo cultural, turismo deportivo, turismo de placer o de recreo, turismo de salud, turismo de negocios: turismo de congresos y convenciones, viajes de incentivos, ferias y exposiciones; turismo naturalista: naturalista suave (soft nature), naturaleza fuerte (hard nature), ecoturismo; entre otros (Quesada, 2010, p. 95).

El turismo cultural, propiamente dicho y entendido como una tipología específica, puede ser definido como aquel turismo cuya motivación principal es aprender, descubrir experiencias y consumir las atracciones y productos culturales materiales e inmateriales locales o del destino visitado. Esos atractivos o productos (materiales, emocionales, espirituales) son los distintivos de la comunidad (Richards, 2018).

Considerando las actividades y no las motivaciones, Calle y García sostienen:

El turismo cultural se basaría en el «uso», «disfrute» y/o «consumo» de elementos del entramado cultural, es decir en la realización de una serie de actividades o prácticas culturales (visita a museos, asistencia a exposiciones, paseos por el casco histórico...) fuera del lugar de residencia habitual. (2010, p. 34)

Por otro lado, la práctica del turismo cultural ha sido asociada tradicionalmente a grupos de élite con conocimientos suficientes para apreciar y valorar las grandes obras artísticas e históricas. Sin embargo, a inicios de los 90 este turismo se vuelve masivo, se abre a un público más amplio (Richards, 2018). El turismo cultural es, por tanto, un segmento de gran crecimiento: según el Report on Tourism and Culture Synergies, el 39\% de todos los arribos del mundo son por turismo cultural (World Tourism Organization, 2018a), lo que representa una gran oportunidad para destinos con diversidad de recursos turísticos culturales, como es el caso del Perú.

El aumento de la demanda del turismo cultural está también producido en parte por la evolución de las condiciones y los modelos del viaje turístico, según los cuáles estamos actualmente en una tendencia hacia estancias más cortas y viajes más frecuentes, fragmentando de esta 
manera, el periodo vacacional de cada persona. Estas estancias más cortas implican visitas más concretas, centradas principalmente en un turismo urbano y cultural. (Esteban \& López, 2008, p. 4)

El turismo cultural, en otro orden de ideas, ha sido identificado históricamente con prácticas poco divertidas, señalado como un turismo aburrido, quizá muy «formal» como para practicarse durante el tiempo libre disponible. En un estudio realizado en México se encontró «que la asociación de un destino con 'cultura' le resta atractivo a un viaje, pues se le vincula con cuestiones escolares, aburrimiento y aridez, mientras que los turistas desean relajarse, divertirse y disfrutar actividades al aire libre» (Amaya, 2006, p. 24).

Frente a la multiplicidad de opciones y actividades, así como a la apertura de lugares cuyos recursos y atractivos son materiales o físicos (incluyéndose ahora lo inmaterial decididamente como parte de la oferta), han emergido otros tipos de actividades, que van desde los vinculados a la gastronomía y religión, hasta los que tienen como fin asistir a festivales, aprendizaje de idiomas, creación, etc., que bien podrían revertir la imagen de un turismo considerado aburrido.

Martorell (2017) plantea tres tipos de factores a tomar en cuenta en el turismo cultural: permanentes, dinámicos y procesales ${ }^{2}$. Los permanentes, referidos al hecho cultural propiamente (patrimonio cultural), son los que fungen de atractivos, la comunidad anfitriona y la infraestructura conexa al hecho cultural que brinda soporte al turismo cultural. Los dinámicos, corresponden al turista cultural, precisamente lo es, mientras está de viaje, «esa temporalidad es un dato clave, porque en la mayor parte de los planes relativos al turismo cultural, se suele tratar al turista como el elemento más importante de la relación» (Martorell, 2017, p. 75). Y los procesales, el tercer

2 El autor cita a Korstange (2013, p. 10), quien a su vez se basa en Santana-Talavera. La cita sirve de soporte para el planteamiento y desarrollo de los componentes involucrados en el turismo cultural, aunque Santana-Talavera se enfoca en el funcionamiento del turismo en general: «El primero se refiere a un elemento dinámico el cual no es otra cosa que la demanda por la cual se movilizan todos los actores dentro del sistema, ya sea por consumo de comodities o bienes con valor agregado. Segundo, tenemos al componente estático lo cual posibilita (en forma de infraestructura) la actividad turística y su posterior ordenamiento territorial. Por último, el componente secuencial, por el cual las partes integradas a un todo se entrelazan para dar origen al sistema (principio de homeóstasis)» (como se citó en Martorell, 2017, p. 74). 
factor, lo integran el desplazamiento (por sí mismo); los gestores del turismo (es decir, quienes posibilitan la práctica del turismo); el encuentro cultural (que para Martorell (2017, p. 75) es la «quinta esencia del viaje cultural»); y las dinámicas posviaje (la experiencia turística).

En este punto, es importante destacar el rol que juegan los factores dinámicos con relación a los permanentes y procesales, al mismo tiempo con la sostenibilidad y respeto por la cultura local. Y es que, si bien suele ponerse al turista (actor temporal) en medio de los esfuerzos en las gestiones turísticas, esta ubicación privilegiada debiera pertenecer a los factores permanentes y consecuentemente a los procesales, pues los primeros, los permanentes, son los que trascienden a la visita y vivirán (comunidad anfitriona y patrimonio cultural) directamente los cambios que pudieran producirse. No es un tema baladí, pues se está hablando de la forma como han vivido y viven las comunidades; de eventualmente, ver modificadas sus costumbres, prácticas cotidianas, lugares (patrimonio cultural en general), los mismos que seguramente le han dado sentido a su vida en comunidad, y por ende, a su vida personal, pues «la identidad personal, la autoimagen de las personas se genera forzosamente en un espacio colectivo y en virtud de un conjunto de asunciones y creencias colectivas, en lo sincrónico y en lo diacrónico» (Massó, 2006, p. 94). Por su parte, los factores procesales, deben concretarse con el cuidado y respeto por los permanentes; es decir, el encuentro cultural, las gestiones y las experiencias deberían estar revestidas de la comprensión por el patrimonio cultural local. Por consiguiente, se necesita un nuevo turista más informado y responsable, para quien debe «hacerse esfuerzos por desarrollar [...] capacidades de disfrute de la experiencia cultural en su mayor autenticidad $»^{3}$ (Martorell, 2017, p. 53), vale decir, «enseñarle a ser turista» (Martorell, como se citó en Alberca, 2018, p. 107).

A propósito del turista cultural, por su comportamiento, Ramos (2007) lo denomina: de inspiración cultural, atraído por la cultura o de motivación cultural (Tabla 1).

3 Palabras dichas durante la conferencia «Las convenciones UNESCO para la protección y salvaguarda del patrimonio cultural. Un marco global para el diseño de políticas públicas» de la Cátedra UNESCO de la Universidad de San Martín de Porres titulada Patrimonio Cultural y Turismo Sostenible, el 14 de mayo de 2018. 


\section{Tabla 1}

Turista cultural según su comportamiento

$\begin{array}{ll}\begin{array}{l}\text { Turista de } \\ \text { inspiración cultural }\end{array} & \begin{array}{l}\text { Elige un destino por su patrimonio cultural, pero } \\ \text { con la intención de realizar la visita una sola vez } \\ \text { en la vida. }\end{array} \\ \begin{array}{l}\text { Turista atraído } \\ \text { por la cultura }\end{array} & \begin{array}{l}\text { Tiene como motivación principal otra que no } \\ \text { sea cultural, sin embargo, no tiene reparos en } \\ \text { acercarse ocasionalmente a lugares históricos. }\end{array} \\ \begin{array}{l}\text { Turista de } \\ \text { motivación cultural }\end{array} & \begin{array}{l}\text { Elige los lugares que visita en función de la } \\ \text { oferta cultural que ofrecen. }\end{array}\end{array}$

Nota: Adaptado de Ramos (2007, p. 68).

El turismo cultural, por la amplitud que el término cultura entraña, es clasificado de diversas maneras, como la que presenta Csapó, que se basa en las particularidades del destino, así como en sus manifestaciones y valores culturales (como se citó en Martorell, 2017, p. 79):

- Turismo patrimonial

- Rutas culturales temáticas

- Turismo en ciudades culturales, tours culturales

- Tradiciones, turismo étnico

- Eventos y festivales de turismo

- Turismo religioso, rutas de peregrinaje

- Cultura creativa y turismo creativo

En esta clasificación, el último punto, el turismo creativo, es un turismo cultural no convencional que promueve la capacidad de crear del visitante, quien toma como referencia la cultura local o de acogida. Es un turismo basado en el patrimonio cultural local, que lo convierte en atractivo y producto turístico (Gonçalves \& Costa, 2018); si se vincula con la definición de turismo cultural, calza con ella de forma precisa, puesto que busca descubrir el patrimonio del visitado por medio de la cocreación. En este punto cabe señalar que el turismo cultural no se centra en «visitas al pasado», sino que incluye la visita y consumo de manifestaciones y espacios -culturales- contemporáneos (Esteban \& López, 2008). 


\section{Un turismo basado en el patrimonio cultural inmaterial}

En el turismo cultural se da la relación entre turismo y cultura. ${ }^{4}$ A propósito del tema, Delgado señala:

La cultura receptora se esfuerza precisamente en ofrecer aquello que le es requerido, de acuerdo con su articulación en un sistema intercultural plenamente sometido a las leyes de la oferta y la demanda. Es lo diferente lo que la sociedad anfitriona sabe que debe exhibir enfáticamente. (como se citó en Tosselli, 2006)

El patrimonio cultural, en tanto, está conformado por el conjunto de bienes y manifestaciones propias de una comunidad, que les permite identificarse, reconocerse, pero también disfrutar y mejorar sus vidas. El patrimonio cultural se debe conjugar en presente, dice Martorell (2017), pues es parte de la herencia recibida, pero que puede ser utilizada en el ahora y ser cuidada al mismo tiempo. El patrimonio cultural puede ser usado para beneficio de sus «dueños» más cercanos, y el turismo (turismo cultural en especial) es una opción. Ese uso, por la naturaleza del patrimonio y su carga física y/o simbólica, deberá ser responsable y sostenible, para cuyo propósito el otro actor, el turista, deberá ser consciente y las administraciones o gestores deberán planificar y gestionar considerando estos aspectos.

En lo relativo al fenómeno del turismo, es necesario recentrar los procesos de planificación en torno a los valores estables, permanentes, continuos y de alta significatividad e impacto, que son los generados por la población local y su patrimonio. Deben hacerse esfuerzos por desarrollar en el turista capacidades de disfrute de la experiencia cultural en su mayor autenticidad, convirtiendo al turismo en un fenómeno de encuentro de valores, respeto y aprendizaje mutuos. (Martorell, 2017, p. 53)

El turismo cultural se ha acercado cada vez más al patrimonio cultural inmaterial y culturas contemporáneas, razón por la cual se ha dado una mayor integración entre el turismo y la economía creativa (Richards, 2018). El

\footnotetext{
4 De manera amplia, el patrimonio cultural está conformado por elementos de la cultura de una comunidad.
} 
patrimonio cultural inmaterial evidentemente carece de materialidad, por lo que su uso y disfrute será diferente. Esto y el contexto de economías creativas, así como las particularidades del turista o consumidor actual (que gusta de mayor protagonismo y experiencia), empujan a que se generen diferentes e innovadoras formas de disfrute del patrimonio cultural inmaterial, entre ellas, el turismo creativo.

Pero la relación entre el turismo y patrimonio cultural no es superficial o restringida a la generación de recursos, como lo señala Martorell:

Y, nuevamente, en nuestros tiempos globales, los ciudadanos del mundo nos hemos convertido en actores del patrimonio y contribuimos constantemente a su definición y redefinición; hemos ampliado, con nuestra práctica de visitas e interpretaciones de lo visitado, a redefinirlo. Hay, por ende, un espacio para el turismo cultural en la determinación de los valores del patrimonio, con lo que esta disciplina se convierte en un área de creciente importancia para las ciencias sociales. $(2017$, p. 26)

Sobre este punto será necesario considerar lo siguiente:

El turismo como actividad organizada y gestionada a nivel micro y macro no puede tomar solo al patrimonio como un recurso sin propiedad específica, sino que, por el contrario, deberá tener en cuenta, y para todo el proceso, a los locales o receptores que son los que finalmente se abren al foráneo. (Alberca, 2018, p. 109)

\section{Definiendo el turismo creativo}

Para Richards y Wilson lo creativo se divide en tres dimensiones: espectáculos creativos, espacios creativos y turismo creativo (como se citó en Richards, 2011).

El turismo creativo es entendido como una extensión del turismo cultural, pero es además, una respuesta al turismo masivo o industrial (Molina, 2016; Richards, 2011), dada su naturaleza creativa y por ende personal e individual. Es ese sentido, Richards (2011) encontró que los aspectos comunes en las definiciones de turismo creativo son: participación activa, experiencias 
auténticas, desarrollo de potencial creativo y desarrollo de habilidades. En otros términos, un turismo dinámico y personal.

Si bien la palabra cultura, tal como encontró Amaya (2006), le resta atractivo al turismo cultural, dada su asociación con prácticas aburridas, el término creativo, se piensa, puede asociarse con dinamismo ${ }^{5}$, con hacer, con movimiento, con actividad, con experimentación; en otras palabras, es posible que el turismo creativo sea atractivo desde el punto de vista promocional, por consecuencia, prometedor para un destino como el Perú, que además cuenta con recursos turísticos para tal fin.

El turismo creativo, para Richards y Reymond, es aquel tipo de turismo que ofrece la oportunidad a los visitantes de desarrollar su potencial creativo, mediante su participación en experiencias de aprendizaje en los destinos visitados; este turismo es una alternativa de desarrollo que propone involucrar a las personas, comunidades y turistas sobre la base del conocimiento local (como se citó en Cavalcanti et al., 2018, p. 272).

Entonces, son los anfitriones o receptores quienes ponen a disposición de los visitantes sus conocimientos, su cultura, su patrimonio cultural inmaterial para que los visitantes cocreen, contribuyendo a la mejora del autoestima de la población local (Guerra, Valduga, \& Tomazzoni, 2015). Ergo, hay cinco categorías de creatividad cotidiana que pueden ser la base del turismo creativo: artesanía, refinamiento cultural, creatividad autoexpresiva, creatividad interpersonal y consumo sofisticado de medios. ${ }^{6}$ Las cinco son basales porque son fáciles de usar y practicar y, por lo tanto, pueden posibilitar la participación activa del visitante (Tan, Kung, \& Luh, 2013).

Por otro lado, debido a que el turismo creativo se sostiene en el patrimonio cultural inmaterial, su acercamiento no debe ser solo utilitario. Las acciones o actividades de interpretación del patrimonio deben ser consideradas en las prácticas turístico creativas, de forma que tengan sentido y sean responsables. Es así que la creatividad, el hacer de los turistas, podría ser considerada como

5 Indagar con qué se asocia el término turismo creativo forma parte del proyecto de investigación del autor del presente artículo.

6 Traducción del texto: Craft, cultural refinement, self-expressive creativity, interpersonal creativity and sophisticated media consumption (Tan et al., 2013, p. 158). 
herramienta de interpretación activa del patrimonio cultural, de modo que su uso enriquezca y permita la comprensión de la manifestación que posibilitó su creación.

La esencia del turismo creativo parece residir en actividades y experiencias relacionadas con la autorealización y la autoexpresión en las que los turistas se convierten en coartistas y cocreadores a medida que desarrollan sus habilidades creativas (Richards, 2011, p. 1237), lo que agrega nuevos valores al consumo del producto turístico, por esa base de cocreación y coparticipación (Guerra et al., 2015), además de ser un turismo que permite la autorrealización y autoexpresión (Gonçalves \& Costa, 2018).

Ahora bien, se ha dicho que el turismo creativo se basa principalmente en el patrimonio cultural inmaterial de una comunidad, lo que no hace que las prácticas creativas se circunscriban solo a las manifestaciones tradicionales, sino que se incluye también las contemporáneas, es decir, aquellas que involucran algunas industrias culturales (teatro, conciertos, festivales, fotografía u otros), sin dejar de lado, lo tradicional (artesanías, alfarería, danzas, literatura u otros) y la creación artística (danza, pintura, escultura, cocina, otros). Es necesario precisar que al hablar de patrimonio cultural inmaterial de una comunidad se hace referencia al declarado formalmente y al que no ha sido reconocido oficialmente por los Estados, pero sí por la población en su práctica diaria, pues lo viven y está vigente entre ellos.

Según Molina (2016) el desarrollo del turismo creativo debe fundamentarse en principios ineludibles, los mismos que se interrelacionan y potencian (Tabla 2).

En el proceso de planificación, gestión y desarrollo del turismo creativo no se debe copiar modelos o experiencias, debido a que cada territorio tiene sus particularidades: su gente reconoce como propias algunas manifestaciones, que otros, en otros lugares, no lo hacen.

El turismo creativo no se basa en fórmulas únicas, en este sentido no es dogmático. Plantea que cada comunidad debe encontrar su camino y darles a sus recursos los usos que aseguren el mejoramiento del bienestar local. Para ello el turismo se concibe como una estrategia y no como un fin. (Molina, 2016, p. 173) 
Tabla 2

Principios del turismo creativo

$\begin{array}{ll}\text { Abundancia } & \begin{array}{l}\text { Disponibilidad de todos los recursos del lugar. } \\ \text { Incluye todas las dimensiones de la realidad } \\ \text { humana y no solo lo vinculado a la dimensión } \\ \text { económica. }\end{array} \\ \text { Sincronicidad } & \begin{array}{l}\text { Vinculación y coordinación de «fuerzas, } \\ \text { capacidades y recursos». }\end{array} \\ \text { Colaboración } & \begin{array}{l}\text { El trabajo colaborativo es más efectivo que el } \\ \text { fragmentado o individual. }\end{array} \\ \text { Ubicuidad o no } & \begin{array}{l}\text { Los recursos para el turismo creativo se } \\ \text { encuentran disponibles libremente, es decir, } \\ \text { por doquier. }\end{array} \\ \text { Recalidad } & \begin{array}{l}\text { Importante por reconexión con raíces locales y } \\ \text { promoción de capacidades del visitante. }\end{array} \\ \text { Comunicación } & \begin{array}{l}\text { Es un proceso de comunicación entre los } \\ \text { integrantes de la «familia de desarrollo», }\end{array} \\ \text { además, con su entorno. }\end{array}$

Nota: Adaptado de Molina (2016, p. 173).

Al planificar y gestionar la atención debe estar puesta en las poblaciones anfitrionas o receptoras, no obstante, es también importante considerar que este turismo, el creativo, transforma al consumidor, pues le da mayor protagonismo al ser él mismo quien crea, agregándole valor al consumo turístico (Guerra et al., 2015, p. 102).

Las «familias de desarrollo» están formadas por socios estratégicos, partes interesadas y turistas de un espacio determinado que se unen para trabajar por el desarrollo turístico (Molina, 2016). 


\section{Conclusiones}

Las características de la sociedad actual ofrecen condiciones para el desarrollo del turismo creativo, erigiéndolo en oportunidad para los destinos turísticos del Perú. Como consecuencia, el turista cultural en general, según sus características, encuentra en el turismo creativo una alternativa.

En el turismo, basado en el uso del patrimonio cultural inmaterial, convergen por el lado del turista, el descubrimiento de este patrimonio, del anfitrión y de la posibilidad de autocrearse experiencias; mientras que, por el lado del anfitrión, un turismo de baja presión o no masivo, que atenúa los potenciales efectos negativos. Con todo, el uso turístico del patrimonio cultural inmaterial debe ser sostenible y responsable, puesto que el contacto entre anfitrión y visitante es directo y cercano.

Finalmente, además de la exposición de ideas de corte teórico-conceptual sobre el turismo creativo, que se efectúa en el presente estudio, es recomendable realizar una investigación sistemática del tema, con trabajos de campo y diseño de productos turísticos especializados.

\section{Referencias}

Alberca, F. (2018). El patrimonio cultural en la formación de los profesionales del turismo en el Perú, 2018. Cultura, 32, 99-122. https://doi.org/10.24265/cultura.2018.v32.05

Amaya, C. (2006). Relaciones entre el turismo y la cultura: turismo cultural y cultura turística en México y en Colima. Estudios sobre las culturas contemporáneas, XII(24), 9-33. Recuperado de http://www.redalyc.org/ articulo.oa?id=31602402

Bezerra, É. D., Melo, M., \& Rocha, A. (2012). El turismo en la sociedad de la información. Un abordaje conceptual sobre el «pos-turismo». Estudios y perspectivas en turismo, 21, 1262-1280. Recuperado de http:/l www.estudiosenturismo.com.ar/PDF/V21/N05/v21n5a11.pdf

Calle, M., \& García, M. (2010). Reflexiones sobre el turismo cultural. La aportación de la cultura en la conformación de flujos turísticos emisores en España y la comunidad de Madrid. Anales de geografía de la Universidad Complutense, 30(2), 31-58. Recuperado de https://search.proquest.com/docview/ 822630037 ? accountid $=14747$

Cavalcanti, D., Medeiros de Araujo, L., \& Guimarães, A. (2018). Oportunidades para o turismo criativo em Alagoas, Brasil. Revista Iberoamericana de Turismo - RITUR, 8, 270-287. https://doi.org/10.2436/20.8070.01.112

Cohen, E. (2005). Principales tendencias en el turismo contemporáneo. Política y Sociedad, 42(1), 11-24. https:/ /doi.org/10.5209/POSO.24128

De Freitas, M., De Sevillha, M., \& Aráujo, A. (2018). Tourism experiences: Core processes of memorable trips. Journal of Hospitality and Tourism Management, 37(September), 11-22. https://doi.org/10.1016/ j.jhtm.2018.08.004 
Esteban, J., \& López, A. (2008). La demanda del turismo cultural y su vinculación con el medio ambiente urbano: los casos de Madrid y Valencia. Madrid: Universidad Complutense de Madrid.

Gonçalves, F., \& Costa, C. (2018). A perceção dos visitantes do território do «Galo de Barcelos»: Destino de Turismo Criativo. Revista Turismo \& Desenvolvimento, 1(27/28), 1177-1194.

Guerra, M., Valduga, V., \& Tomazzoni, E. (2015). Turismo criativo e desenvolvimento da oferta turística do cluster do Vale dos Vinhedos (RS, Brasil). Investigaciones Turísticas, 10, 90-116.

Khatchikian, M. (2000). Historia del Turismo. Lima: Universidad de San Martín de Porres.

Martorell, A. (2017). Turismo cultural. Reflexiones para un encuentro sostenible entre turismo y cultura. Lima: Universidad de San Martín de Porres.

Massó, E. (2006). La identidad cultural como patrimonio inmaterial: Relaciones dialécticas con el desarrollo. Theoria, 15(1), 89-99. Recuperado de http://web.b.ebscohost.com/ehost/pdfviewer/pdfviewer?sid=bcdd8f814cda-4522-a89e-e4f4de9ab94b\%40sessionmgr113\&vid=28\&hid=105

Molina, S. (2016). Turismo creativo. Investigaciones Turísticas, 6, 170-173.

Quesada, R. (2010). Elementos del turismo. Recuperado de http://books.google.com.pe/books?id=RdrDv_52Lm $Y C \&$ printsec=frontcover\&dq=teoria+turismo\&source=bl\&ots=WMTHLqHTtI\&sig=xm Rw1uc8dh4bW0 gewNv4tMMxjUI\&hl=es\&sa=X\&ei=97JjUJfSJY-68wSxilGQDA\&ved= 0CDIQ6AEwAQ\#v=onepage\&q=teoria turismo\&f=false

Ramos, M. (2007). El turismo cultural, los museos y su planificación. Gijón: Trea, S. L.

Richards, G. (2011). The State of the Art. Annals of Tourism Research, 38(4), 1225-1253. https://doi.org/10.1016/ j.annals.2011.07.008

Richards, G. (2018). Cultural tourism: A review of recent research and trends. Journal of Hospitality and Tourism Management, 36, 12-21. https://doi.org/10.1016/j.jhtm.2018.03.005

Tan, S., Kung, S.-F., \& Luh, D.-B. (2013). A model of «Creative experience» in creative tourism. Annals of Tourism Research, 41, 153-174. https://doi.org/10.1016/j.annals.2012.12.002

Tosselli, C. (2006). Algunas reflexiones sobre el turismo cultural. PASOS, Revista de Turismo y Patrimonio Cultural, 4(2), 175-182. Recuperado de http://www.pasosonline.org/Publicados/4206/PS040206.pdf

Urry, J. (2004). La mirada del turista. Lima: Escuela Profesional de Turismo y Hotelería, Universidad de San Martín de Porres.

Valdez, R. (2002). Turismo cultural: La experiencia mexicana. En J. García y M. Poyatao (Eds.), La función social del patrimonio histórico: El turismo cultural (pp. 83-104). Recuperado de https://books.google.com.pel books?id=8_nfnBp4Rg4C\&printsec=frontcover\&dq=turismo+cultural\&source=bl\&ots=0ZhvlZm3wW\&sig=dKgo1_2amuE70BJRdwkIVZDpY4\&hl=es\&sa=X\&ei=ndxjJM3bK_CM0QHDIYGIDA\&ved=0CDgQ6AEwAw\#v=onepage\&q =turismo cultural\&f=false

World Tourism Organization. (2018a). Tourism and Culture Synergies. https://doi.org/10.18111/9789284418978 World Tourism Organization. (2018b). UNWTO Annual Report 2017. Madrid: World Tourism Organization. 